\title{
A Modified Outside-in Suture Technique for Repair of the Middle Segment of the Meniscus Using a Spinal Needle
}

\author{
Jin-Ho Cho, MD \\ Department of Orthopaedic Surgery, Inje University Ilsan Paik Hospital, Goyang, Korea
}

\begin{abstract}
Introduction: Several techniques have been used for arthroscopic repair of middle segment, posteromedial or posterolateral corner tears of the meniscus. One of the commonly used methods is the inside-out double arm needle technique.

Surgical Technique: We have developed a vertical mattress absorbable suture technique. It is easy to perform with a small sized skin incision for knot tying. This technique just necessitates 1 or 2 spinal needles for repair.

Materials and Methods: Between March 2010 and February 2012, 20 menisci were treated by this technique, a modified method of the outside-in vertical meniscal repair using a spinal needle and No. 2 PDS absorbable suture material. Evaluation of clinical results was done using the Lysholm score.

Results: The mean preoperative Lysholm score was 63.9 and the mean postoperative score was 97.3. A second look arthroscopy was performed in 13 knees $(65 \%)$ and the repair sites were well healed.

Conclusions: We recommend this method as an alternative technique for repair of the middle segment, posteromedial or posterolateral corner of the meniscus.
\end{abstract}

Keywords: Meniscus, Middle segment, Tear, Modified outside-in suture

\section{Introduction}

Numerous surgical techniques have been developed for meniscal repair: all-inside, inside-out, outside-in, and modifications of these techniques. Each technique poses different advantages and disadvantages. Thus, a repair technique is chosen by a surgeon depending on the location and pattern of a meniscal injury ${ }^{11}$. The inside-out technique uses double-armed sutures passed through arthroscopic cannulas and then tied directly over the

Received April 10, 2013; Revised (1st) May 14, 2013; (2nd) July 11, 2013; (3rd) August 1, 2013; Accepted August 5, 2013

Correspondence to: Jin-Ho Cho, MD

Department of Orthopaedic Surgery, Inje University Ilsan Paik Hospital, 170 Juhwa-ro, Ilsanseo-gu, Goyang 411-706, Korea

Tel: +82-31-910-9733, Fax: +82-31-910-7967

E-mail: osd07@paik.ac.kr

This is an Open Access article distributed under the terms of the Creative Commons Attribution Non-Commercial License (http://creativecommons.org/licenses/by-nc/3.0/) which permits unrestricted non-commercial use, distribution, and reproduction in any medium, provided the original work is properly cited. joint capsule. This technique has several disadvantages. One is the horizontal suture for peripheral tears of the meniscus. Second is the double arm needle technique directed to a far posterolateral or posteromedial side. It may cause neurovascular injuries during procedure. Third is foreign body reaction caused by nonabsorbable double arm needle suture materials ${ }^{2}$. The outside-in technique is most applicable to tears in the anterior and middle segments of the meniscus. But, this technique is horizontal suture and uses a nonabsorbable suture material ${ }^{3)}$, which is different from our technique. The all-inside repair technique is indicated for unstable, vertical-longitudinal tears of the posterior horns of the meniscus. When repairing a meniscal tear, an adequate approximation of torn surfaces with firm fixation is important for yielding an optimal healing. For repair of a middle segment, posteromedial or posterolateral corner tear, a meniscal fixator or the inside-out double arm needle technique is considered effective $^{3)}$. However, with a meniscal fixator, it is not only difficult to attain good coaptation between torn surfaces, the holding power is expected relatively weak. We introduce a modified outside-in vertical suture technique with a spinal needle that provides a safe 
and vertically oriented suture with good coaptation and strong holding power. Therefore, we believe this technique could be effective for repairing most parts of the meniscus, especially for the middle segment, posteromedial or posterolateral corner injuries.

\section{Surgical Technique}

The patient is positioned for standard knee arthroscopy. This

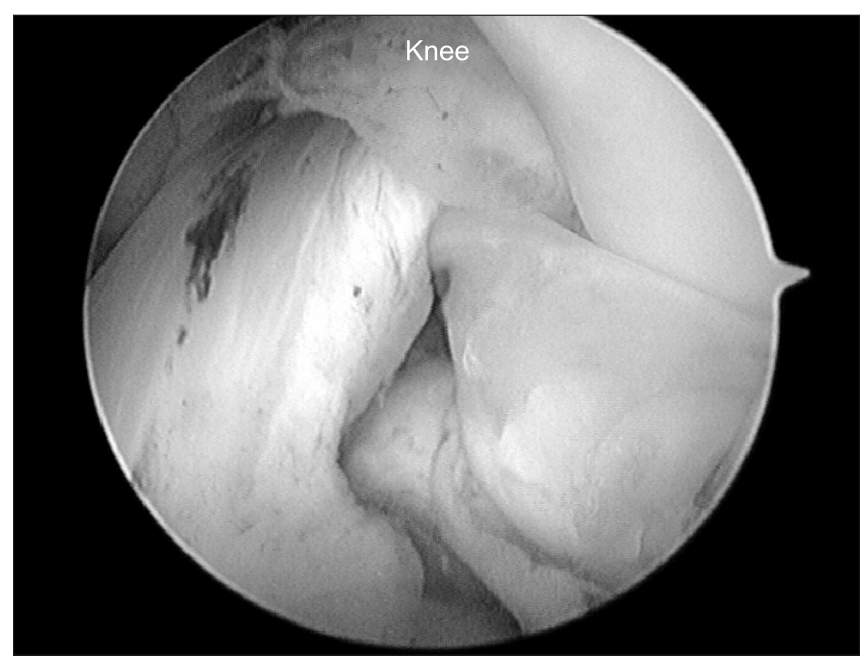

Fig. 1. Arthroscopic view of a lateral meniscus bucket-handle tear visualized through the anterolateral portal of the left knee. technique for lateral meniscus bucket-handle tear of the left knee (Fig. 1) requires an 18-gauge spinal needle, a suture retriever, and an absorbable suture material. After confirmation of a tear, the tear site is debrided with a $3.5 \mathrm{~mm}$ full-radius motorized shaver (Dyonics; Smith \& Nephew, Andover, MA, USA) and a rasp to encourage healing through the portal. At first, the posterior horn tear site is repaired with 2 or 3 all inside sutures via a posterolateral portal. Then, the middle horn is repaired using modified outside-in vertical sutures. On the skin near the middle portion of the tear site, the 18-gauge spinal needle first penetrates the capsular portion, crosses the tear, and then exits the tibial surface of the inner fragment of the middle horn of the lateral meniscus. This procedure can be made easier by the use of a grasper introduced through the far anteromedial portal to provide counter pressure on the torn meniscus. No. 2 PDS (Ethicon, Somerville, NJ, USA) suture material is advanced through the spinal needle (Fig. 2). The procedure of pulling out the suture tip via the anteromedial portal should be performed carefully so as not to cut the suture material by the beveled needle tip. Then, the needle is pulled back out of the skin. A second needle is reinserted downward and penetrated through the tibial side of the tear of menisus. No. 0 Maxon (Covidien, Mansfield, MA, USA) suture material is advanced through the spinal needle (Fig. 3). The procedure of pulling out the suture tip via the anteromedial portal using a suture retriever should be performed carefully so as not to cut the
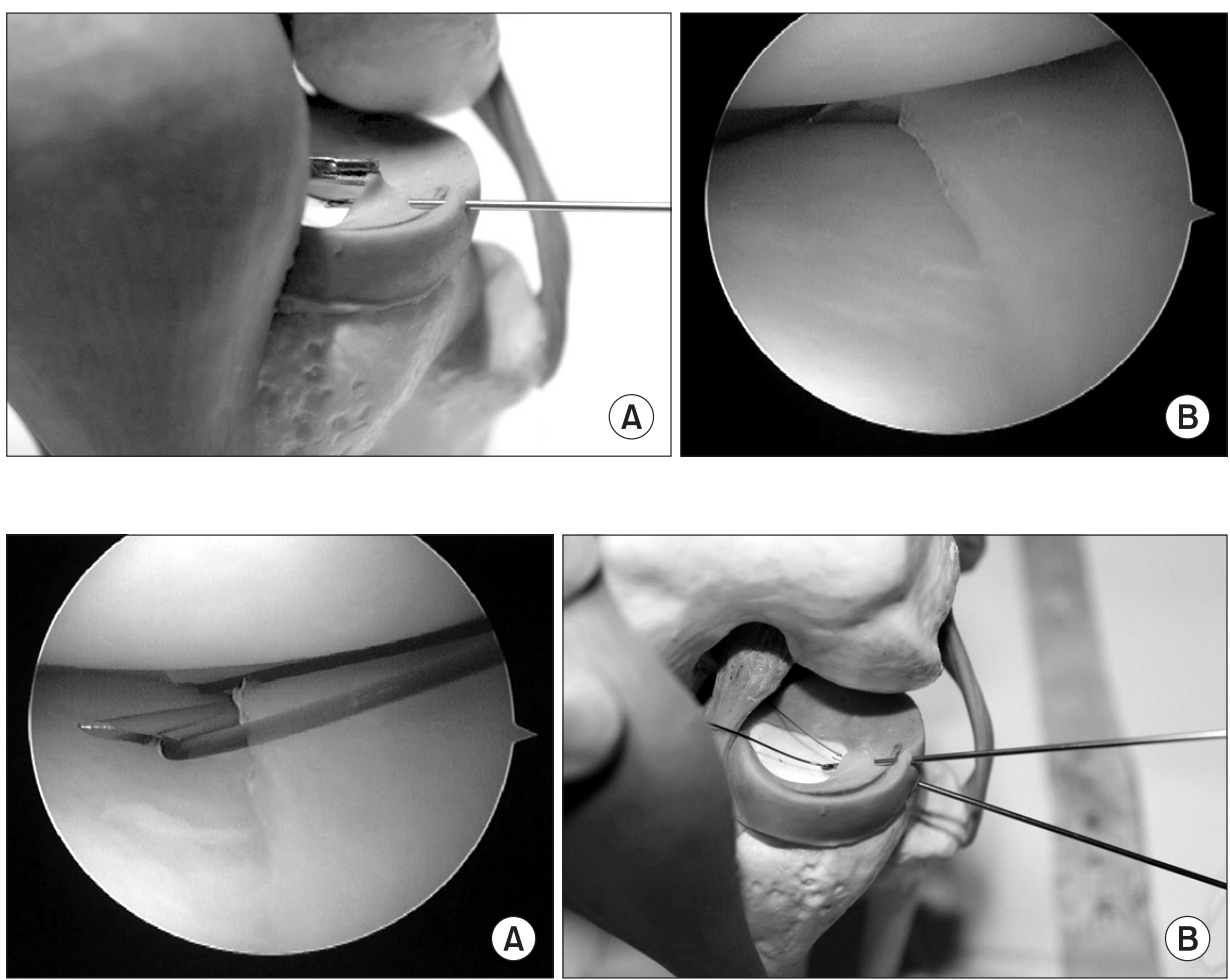

Fig. 2. (A) An 18-gauge spinal needle first penetrates the capsular portion, crosses the tear, and then exits the tibial surface of the inner fragment of the middle segment of the lateral meniscus in the bone model. (B) Arthroscopic image of No. 2 PDS suture material advanced through the spinal needle.

Fig. 3. (A) The arthroscopic view from the anterolateral portal shows the second needle is reinserted downward and penetrates through the tibial side of the tear of the menisus. No. 0 Maxon suture material is advanced through the spinal needle. (B) A bone model showing the same procedure. 

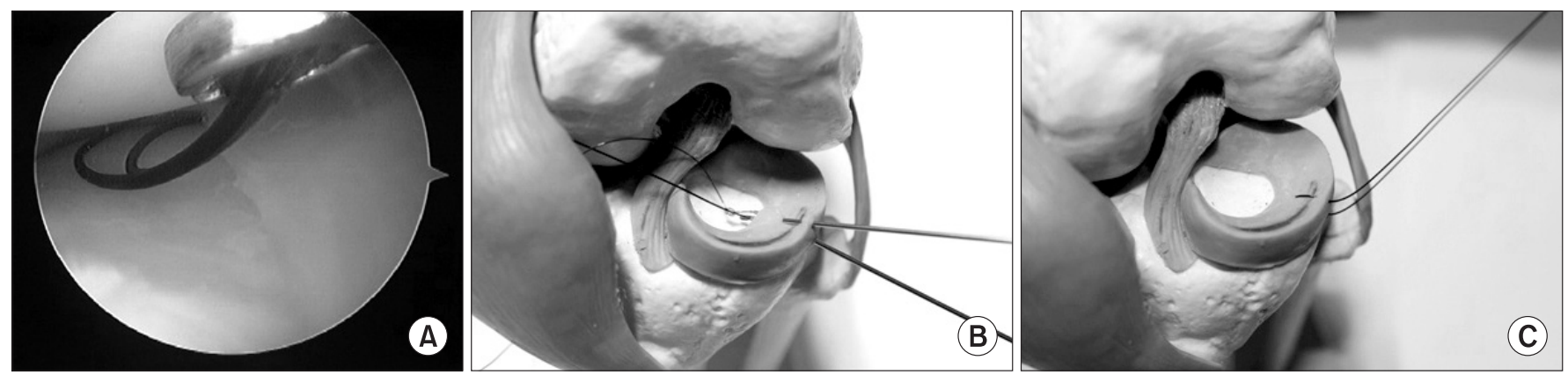

Fig. 4. (A) The arthroscopic view from the anterolateral portal shows the procedure of pulling out the suture tip via the anteromedial portal using a suture retriever. (B) After tying a knot of Maxon holding the tibial end of PDS outside the knee joint, the other side of Maxon is pulled out of the skin of the mid portion of the joint for retrieving into the joint of the tibial side of PDS. (C) The suture then becomes a vertical mattress stitch ready to be tied on the bone model.
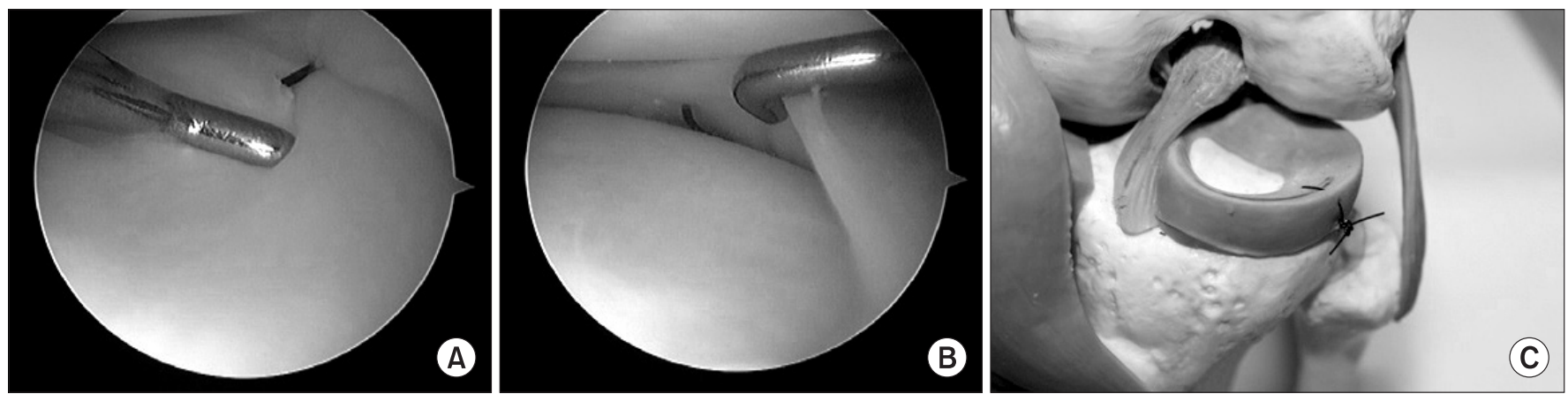

Fig. 5. (A, B) Arthroscopic views from the anterolateral portal show the suture is oriented in a vertical fashion. (C) The bone model shows completion of the vertical absorbable suture.

suture material by the beveled needle tip. After tying a knot of the Maxon holding the tibial end of the PDS outside the knee joint, the other side of the Maxon is pulled out of the skin at the mid portion of the joint for being retrieved into the joint of the tibial side of the PDS. The suture, then, becomes a vertical mattress stitch ready to be tied (Fig. 4). The same procedure is repeated as often as necessary to perform stable meniscal repair. An approximately $1-\mathrm{cm}$ skin incision is made in the region corresponding to a meniscal tear, both suture ends are tightened to adequately tension the reduced meniscus without any soft tissue interposition via direct arthroscopic visualization and the first knot is made tightly (Fig. 5). After knot tying, tension is checked with a probe via arthroscopic visualization and then the second and third knots are made. With this method, a suture can be oriented in a vertical fashion, thus providing an adequate contact between torn surfaces while maintaining firm fixation.

\section{Materials and Methods}

Between March 2010 and February 2012, arthroscopic meniscal repair was performed using our technique in 20 knees. Indications for meniscal repair included medial (6 cases) or lateral (14 cases) meniscus middle segment tears in the red-red zone or redwhite zone, longitudinal tears, single tears, tears in stable or stabilizable knees, young age ( $<50$ years).

Evaluation of clinical results was done using the Lysholm score. Patient's average age was 34 years (range, 15 to 46 years). The follow-up period was 14 months (range, 12 to 23 months). Causes of injury were slip down in 14 cases and traffic accident in 6 cases.

After surgery, a cylinder leg splint was applied for 1 week in a fully extended position and a limited-motion brace was subsequently applied to control motions for 6-8 weeks. Patients remained non-weight bearing on crutches for $4-6$ weeks. They were instructed to perform quadriceps muscle exercises as well as straight leg raise exercises several times per day. Up to $90^{\circ}$ of active range of motion was allowed after the first 4 postoperative weeks, which was gradually increased to up to $135^{\circ}$ of flexion. Full flexion and squatting were allowed 3 months postoperatively. They returned to exercise 6 months postoperatively. 


\section{Results}

The mean preoperative Lysholm score was 63.9 and the mean postoperative score was 97.3.

A second look arthroscopy was performed in 13 knees (65\% of the total 20 knees). The repaired sites appeared well-healed and patients' symptoms were improved. No complications related to this modified outside-in arthroscopic meniscal repair procedure occurred.

\section{Discussion}

The goal of the treatment of torn menisci was symptomatic relief through open total meniscectomy in the past. However, it has been shifted towards preservation of meniscal functions as well as symptomatic improvement ${ }^{4}$. Ahn et al. ${ }^{2}$ reported a modified inside-out technique using a suture hook, a flexible looped needle and a zone specific cannula. This technique is advantageous over the classic inside-out technique in that it allows for a vertical suture and use of an absorbable suture, such as PDS. However, this technique has some disadvantages. First, if the joint space is narrow, suture hook handling becomes difficult, and an articular cartilage injury may occur during the procedure. Second, it also requires the use of a double arm cannula: when a double arm cannula is directed to a far posterolateral or posteromedial side, neurovascular injuries may occur. Third, flexible looped needles are weak, so they are often broken during procedure. Abdelkafy et al. ${ }^{3)}$ used the outside-in suture technique. Compared to our technique, this technique is for a horizontal suture, not a vertical suture. It also uses two monofilament non-absorbable 2-0 suture materials $s^{3)}$ that remain in the joint after healing of a tear site. It can irritate articular cartilage or cause a foreign body reaction. Rodeo $^{5)}$ reported that the outside-in technique was especially useful for suturing the middle segment of the meniscus. However, with the conventional outside-in suture technique, an additional skin incision is required and subcutaneous knot irritation may occur. Kelly and Ebrahimpour ${ }^{6}$ reported on the evolution of synovitis and femoral articular cartilage injury after arthroscopic outside-in repair of the medial meniscus using Mulberry knots (knot-end sutures). Horizontal sutures are commonly made with the inside-out technique due to the difficulty of making vertically oriented sutures. However, according to Rimmer et al. ${ }^{7}$, the failure strength of the horizontal suture $(29.3 \mathrm{~N})$ that parallels the alignment of meniscal collagen fiber is significantly less than that of the vertical suture $(67.4 \mathrm{~N})$ that is perpendicular to the alignment of the collagen fiber meniscus. Although the double-arm needle technique that involves passages of two needles on the femoral side and two needles on the tibial side of the meniscus for vertically oriented double sutures has been introduced, passage of four needles in a parallel fashion is a technically challenging task ${ }^{8}$. Suturing with a bioabsorbable fixator is a convenient method with advantages of shortened operation time, less neurovascular injury, absence of postoperative limitation in range of motion associated with capsular adhesion, and no need for an additional skin incision ${ }^{3)}$. However, to secure adequate fixation, at least $3 \mathrm{~mm}$ of the remnant meniscus should be intact ${ }^{9}$. If a posteromedial injury is present in the meniscocapsular junction, adequate fixation might not be obtained and subsequent fixation failure could occur due to inferior migration of the capsule meniscus ${ }^{9)}$.

Our modified outside-in suture technique is simple and safe and yields more accurate anatomic reduction and stronger fixation strength by tying the vertically oriented absorbable suture material above and below the meniscus. A vertically oriented suture has been demonstrated to have greater pull-out strength than a horizontally oriented suture, because the vertical orientation can capture the circumferentially oriented collagen fibers of the meniscus more effectively ${ }^{10)}$. In addition, it can hold the meniscus symmetrically, yield anatomical reduction and provide good coaptation ${ }^{11}$. In case of a long longitudinal tear or a bucket handle tear, attainment of anatomic reduction through one or two modified outside-in sutures followed by additional fixation with a bioabsorbable meniscal fixator or the conventional insideout double arm needle technique could shorten the operation time and yield better clinical results. Firm fixation by a vertical suture and increasing the contact surface of the torn pieces by anatomic reduction make the modified outside-in technique a superior method in treating tears in most portions of the anterior horn, middle segment, posteromedial or posterolateral corner of the medial or lateral meniscus. Disadvantages include the risk of articular cartilage injury caused by spinal needles, technical difficulty in narrow joint spaces especially in cases of medial meniscus injuries of the varus knee.

\section{Conclusions}

This modified outside-in suture technique has several advantages compared to the previous outside-in and inside-out techniques. First, it is easy to perform and cosmetically superior in treating anterior horn, middle segment, posteromedial or posterolateral corner meniscal injuries. Second, this technique allows for vertical mattress absorbable sutures as opposed to the previ- 
ous outside-in method. Third, this technique just necessitates 1 or 2 spinal needles for repair.

Therefore, we recommend this technique as a new alternative repairing method for tears in most portions of the anterior horn, middle segment, posteromedial or posterolateral corner of the medial or lateral meniscus.

\section{Conflict of Interest}

No potential conflict of interest relevant to this article was reported.

\section{References}

1. Cho JH. Arthroscopic all-inside repair of anterior horn tears of the lateral meniscus using a spinal needle. Knee Surg Sports Traumatol Arthrosc. 2008;16:683-6.

2. Ahn JH, Wang JH, Oh I. Modified inside-out technique for meniscal repair. Arthroscopy. 2004;20 Suppl 2:178-82.

3. Abdelkafy A, Aigner N, Zada M, Elghoul Y, Abdelsadek H, Landsiedl F. Two to nineteen years follow-up of arthroscopic meniscal repair using the outside-in technique: a retrospective study. Arch Orthop Trauma Surg. 2007;127:245-52.

4. Lee BS, Kim JM, Sohn DW, Bin SI. Review of meniscal allograft transplantation focusing on long-term results and evaluation methods. Knee Surg Relat Res. 2013;25:1-6.

5. Rodeo SA. Arthroscopic meniscal repair with use of the outside-in technique. Instr Course Lect. 2000;49:195-206.

6. Kelly JD 4th, Ebrahimpour P. Chondral injury and synovitis after arthroscopic meniscal repair using an outside-in mulberry knot suture technique. Arthroscopy. 2004;20:e49-52.

7. Rimmer MG, Nawana NS, Keene GC, Pearcy MJ. Failure strengths of different meniscal suturing techniques. Arthroscopy. 1995;11:146-50.

8. Rubman MH, Noyes FR, Barber-Westin SD. Arthroscopic repair of meniscal tears that extend into the avascular zone: a review of 198 single and complex tears. Am J Sports Med. 1998;26:87-95.

9. Seil R, Rupp S, Dienst M, Mueller B, Bonkhoff H, Kohn DM. Chondral lesions after arthroscopic meniscus repair using meniscus arrows. Arthroscopy. 2000;16:E17.

10. Post WR, Akers SR, Kish V. Load to failure of common meniscal repair techniques: effects of suture technique and suture material. Arthroscopy. 1997;13:731-6.

11. Ahn JH, Wang JH, Yoo JC, Kim SK, Park JH, Park JW. The modified outside-in suture: vertical repair of the anterior horn of the meniscus after decompression of a large meniscal cyst. Knee Surg Sports Traumatol Arthrosc. 2006;14:128891. 